\title{
Old Mechanics, Gravity, Electromagnetics and Relativity in One Theory: Part I
}

\author{
Abed El Karim S. Abou Layla \\ Independent Researcher, Gaza City, Palestine \\ Email: a.k.aboulayla@gmail.com
}

How to cite this paper: Abou Layla, A.K. (2018) Old Mechanics, Gravity, Electromagnetics and Relativity in One Theory: Part I. Journal of High Energy Physics, Gravitation and Cosmology, 4, 529-540. https://doi.org/10.4236/jhepgc.2018.43031

Received: April 1, 2018

Accepted: July 23, 2018

Published: July 26, 2018

Copyright $\odot 2018$ by author and Scientific Research Publishing Inc. This work is licensed under the Creative Commons Attribution International License (CC BY 4.0).

http://creativecommons.org/licenses/by/4.0/

\begin{abstract}
This paper research is the first part of the scientific theory that seeks to unify the sciences of physics with the minimal number of mathematical formulas as possible. We will prove that all equations of forces in nature can be concised in two mathematical formulas, no difference between gravitational or electrical forces or any other type of Types of conventional forces, and through the equivalence of the concepts of matrix and vector, in this theory we will be linking the four-dimensional forces equations with the classical physics as an introduction to connect the rest of the physical sciences.
\end{abstract}

\section{Keywords}

Equations of Force, Gravity, Electromagnetism, Khromatic Theory, Maxwell's Equations, Relativity

\section{Introduction}

Numerous of recent books in physics discourse the summarizing of Maxwell's four equations into two equations, without addressing the possibility to generalize this concept to the rest of the forces, and work to link them with classical physics, which is the goal of publishing this research.

Whereas we cannot link all the physical sciences in one theory, unless the base upon which this theory was built represents a good and common ground for all of these sciences, So at the beginning will get to know some mathematical concepts (for example the relationship between the matrix and the vector) in a new and concise manner, with the remarks that we will deliberately ignore some proofs and details of those concepts to shorten the pages of this research that will exceed tens of pages.

In this part of the theory we will prove that all equations of force in nature 
belong basically to two basic mathematical formulas, on the Figure $c \times \vec{F}$ and $c \cdot \vec{F}$, including Lagrange equation, which will we address in the coming research, and the results obtained in this research will be applied only to the electrical and magnetic forces, with no other ones, since these forces are the most prominent in the books of physics.

In the next parts we will explore the possibility of combining the theories of General Relativity and Quantum Mechanics with this theory in the minimal mathematical relationships as possible.

However, the purpose of publishing this paper can be summarized as follows

1) Introducing new mathematical ideas and concepts which will help to unify physics;

2) Unification of the physical sciences with as few equations as possible (in this part, most of the forces known as only two forms);

3) Linking modern physical science with ancient physics without resorting to any hypotheses (such as the stability of the speed of light in the theory of relativity).

\section{Basic Notions}

\subsection{A.E Filed}

In this paper, the space $\mathbb{R}^{n, m}$ is called A.E space in $\mathrm{n}$-dimensional with m-index filed, in this case. We suppose that the m-index filed vector $\vec{F}$ in $\mathbb{R}^{n, m}$ is defined by

$$
\vec{F}=F_{i j \cdots m} \boldsymbol{v}^{i j \cdots m}
$$

where

$\boldsymbol{v}^{i j \cdots m}$ is a complex orthogonal unit, that is defined by setting

$$
\boldsymbol{v}^{i j \cdots m}=e^{i} \times e^{j} \times \cdots \times e^{m}
$$

$e^{0}, e^{1}, \cdots, e^{n-1}$ are unit vectors in the $x^{0}, x^{1}, \cdots, x^{n-1}$ directions.

In general, the space of two vectors $\boldsymbol{C} \in \mathbb{R}^{n, m}$ and $\vec{F} \in \mathbb{R}^{n, \rho}$ is defined as $\mathbb{R}^{n, m, \rho}$ in $n$-dimensional with $m, \rho$ mix-filed.

\subsection{Theory}

"The cross product of a set of vectors in any specified space equal to the Determinant of these vectors"

It mean that if

$\boldsymbol{A}_{1}=a_{1, j} e^{j}, \boldsymbol{A}_{2}=a_{2, j} e^{j}, \cdots, \boldsymbol{A}_{i}=a_{i, j} e^{j}$ with $i=1,2, \cdots, m ; j=0,1,2, \cdots, n$. And,

$D=A_{1} \times A_{2} \times \cdots \times A_{m}$, then

$$
\boldsymbol{D}=\left|\begin{array}{cccc}
e^{0} & e^{1} & \cdots & e^{n} \\
a_{1,0} & a_{1,1} & \cdots & a_{1, n} \\
a_{2,0} & a_{2,1} & \cdots & a_{2, n} \\
\vdots & \vdots & \ddots & \vdots \\
a_{m, 0} & a_{m, 1} & \cdots & a_{m, n}
\end{array}\right|
$$




\subsection{Determinants and Dual Determinants}

\subsubsection{The Main Determinant}

The main determinant of $(m+1) \times(n)$ matrix has been defined. Denote by $F_{i_{1} i_{2} \cdots i_{m}}$ the sub determinant of the $(m) \times(m)$ matrix obtained from D by deleting the first row and choosing the $i_{1}, i_{2} \cdots, i_{m}$-columns. Then, the main determinant of matrix denote as

$$
\operatorname{det} \boldsymbol{D}=F_{01 \cdots m} \boldsymbol{v}^{01 \cdots m}+\cdots+F_{i_{1} i_{2} \cdots i_{m}} \boldsymbol{v}^{i_{1} i_{2} \cdots i_{m}}+\cdots+F_{n-m+1, \cdots, n} \boldsymbol{v}^{n-m+1, \cdots, n}
$$

where

$m$ : the number of the vectors in the group;

$i_{1}, i_{2} \cdots, i_{m}$ - the numbers of columns which has been chosen.

$$
\boldsymbol{v}^{i_{1} i_{2} \cdots i_{m}}=e^{i_{1}} \times e^{i_{2}} \times \cdots \times e^{i_{m}}
$$

\subsubsection{The Dual Determinant of Matrix Denote as}

$$
\text { dualdet } \overline{\boldsymbol{D}}=\bar{F}_{01 \cdots m} \boldsymbol{v}^{01 \cdots m}+\cdots+\bar{F}_{i_{1} i_{2} \cdots i_{m}} \boldsymbol{v}^{i_{1} i_{2} \cdots i_{m}}+\cdots+\bar{F}_{n-m+1, \cdots, n} \boldsymbol{v}^{n-m+1, \cdots, n}
$$

and the dual sub determinant $\bar{F}$ define by equation,

$$
\bar{F}_{i_{1} i_{2} \cdots i_{m}}=F_{m+1, m+2, \cdots, n}
$$

where

$m+1, m+2, \cdots, n$ - the numbers of columns which has not been chosen.

$i_{1} i_{2} \cdots i_{m}$ or $m+1, m+2, \cdots, n$ denotes the cyclic permutation symmetry.

Let, $\boldsymbol{A}=a_{0} e^{0}+a_{1} e^{1}+a_{2} e^{2}+a_{3} e^{3}: \quad \boldsymbol{B}=b_{0} e^{0}+b_{1} e^{1}+b_{2} e^{2}+b_{3} e^{3}$, and $\vec{F}=\boldsymbol{A} \times \boldsymbol{B}$, Then

$$
\begin{aligned}
& \vec{F}=\operatorname{det} \boldsymbol{D}=\left|\begin{array}{llll}
e^{0} & e^{1} & e^{2} & e^{3} \\
a_{0} & a_{1} & a_{2} & a_{3} \\
b_{0} & b_{1} & b_{2} & b_{3}
\end{array}\right| \\
& \operatorname{det} \boldsymbol{D}=\left|\begin{array}{cccc}
e^{0} & e^{1} & e^{2} & e^{3} \\
a_{0} & a_{1} & a_{2} & a_{3} \\
b_{0} & b_{1} & b_{2} & b_{3}
\end{array}\right| e^{0} \times e^{1}+\left|\begin{array}{cccc}
e^{0} & e^{1} & e^{2} & e^{3} \\
a_{0} & a_{1} & a_{2} & a_{3} \\
b_{0} & b_{1} & b_{2} & b_{3}
\end{array}\right| e^{0} \times e^{2} \\
& +\left|\begin{array}{cccc}
e^{0} & e^{1} & e^{2} & e^{3} \\
a_{0} & a_{1} & a_{2} & a_{3} \\
b_{0} & b_{1} & b_{2} & b_{3}
\end{array}\right| e^{0} \times e^{3}+\left|\begin{array}{cccc}
e^{0} & e^{1} & e^{2} & e^{3} \\
a_{0} & a_{1} & a_{2} & a_{3} \\
b_{0} & b_{1} & b_{2} & b_{3}
\end{array}\right| e^{1} \times e^{2} \\
& +\left|\begin{array}{cccc}
e^{0} & e^{1} & e^{2} & e^{3} \\
a_{0} & a_{1} & a_{2} & a_{3} \\
b_{0} & b_{1} & b_{2} & b_{3}
\end{array}\right| e^{1} \times e^{3}+\left|\begin{array}{cccc}
e^{0} & e^{1} \cdots & e^{2} & e^{3} \\
a_{0} & a_{1} & a_{2} & a_{3} \\
b_{0} & b_{1} & b_{2} & b_{3}
\end{array}\right| e^{2} \times e^{3} \\
& \vec{F}=\left|\begin{array}{cc}
a_{0} & a_{1} \\
b_{0} & b_{1}
\end{array}\right| e^{0} \times e^{1}+\left|\begin{array}{cc}
a_{0} & a_{2} \\
b_{0} & b_{2}
\end{array}\right| e^{0} \times e^{2}+\left|\begin{array}{cc}
a_{0} & a_{3} \\
b_{0} & b_{3}
\end{array}\right| e^{0} \times e^{3} \\
& +\left|\begin{array}{cc}
a_{1} & a_{2} \\
b_{1} & b_{2}
\end{array}\right| e^{1} \times e^{2}+\left|\begin{array}{cc}
a_{1} & a_{3} \\
b_{1} & b_{3}
\end{array}\right| e^{1} \times e^{3}+\left|\begin{array}{cc}
a_{2} & a_{3} \\
b_{2} & b_{3}
\end{array}\right| e^{2} \times e^{3} \\
& =\left|\begin{array}{ll}
a_{\mu} & a_{\gamma} \\
b_{\mu} & b_{\gamma}
\end{array}\right| e^{\mu} \times e^{\gamma}=F_{\mu \gamma} v^{\mu \gamma}
\end{aligned}
$$


Thus,

$$
\vec{F}=F_{01} \boldsymbol{v}^{01}+F_{02} \boldsymbol{v}^{02}+F_{03} \boldsymbol{v}^{03}+F_{12} \boldsymbol{v}^{12}+F_{13} \boldsymbol{v}^{13}+F_{23} \boldsymbol{v}^{23}=F_{i j} \boldsymbol{v}^{i j}
$$

where, $F_{\mu \gamma}=\left|\begin{array}{ll}a_{\mu} & a_{\gamma} \\ b_{\mu} & b_{\gamma}\end{array}\right|=\left(a_{\mu} b_{\gamma}-a_{\gamma} b_{\mu}\right)$.

\section{For example:}

let, $\boldsymbol{A}=5 e^{0}+7 e^{1}-2 e^{2}+13 e^{3}: \quad \boldsymbol{B}=3 e^{0}-11 e^{1}+9 e^{2}+17 e^{3}$.

Then, from above equation we get

$$
\begin{aligned}
\boldsymbol{A} \times \boldsymbol{B} & =\left|\begin{array}{cccc}
e^{0} & e^{1} & e^{2} & e^{3} \\
5 & 7 & -2 & 13 \\
3 & -11 & 9 & 17
\end{array}\right| \\
& =-76 \boldsymbol{v}^{01}+51 \boldsymbol{v}^{02}+46 \boldsymbol{v}^{03}+41 \boldsymbol{v}^{12}+262 \boldsymbol{v}^{13}-151 \boldsymbol{v}^{23}
\end{aligned}
$$

The magnitude or length of the vector $\boldsymbol{A} \times \boldsymbol{B}$ in $\mathbb{R}^{4,2}$ is defined as

$$
|\boldsymbol{A} \times \boldsymbol{B}|=\sqrt{(-76)^{2}+(51)^{2}+(46)^{2}+(41)^{2}+(262)^{2}+(-151)^{2}}=321.8991
$$

From dot product, we get

$\boldsymbol{A} \cdot \boldsymbol{B}=|\boldsymbol{A}||\boldsymbol{B}| \cos \alpha:|\boldsymbol{A} \times \boldsymbol{B}|=|\boldsymbol{A}||\boldsymbol{B}| \sin \alpha$. Thus

$$
\begin{gathered}
\cos \alpha=\frac{\boldsymbol{A} \cdot \boldsymbol{B}}{|\boldsymbol{A}||\boldsymbol{B}|}=\frac{141}{\sqrt{247} \sqrt{500}}=0.401223 \\
|\boldsymbol{A} \times \boldsymbol{B}|=|\boldsymbol{A}||\boldsymbol{B}| \sin \alpha=\sqrt{247} \sqrt{500}(0.91598)=321.8991
\end{gathered}
$$

The last equation equals the length of the vector in $\mathbb{R}^{4,2}$.

\subsubsection{Calculate the Dual to $\vec{F}$}

From (2.1), we can define the dual vector $\overrightarrow{\vec{F}}$ by equation

$$
\overrightarrow{\bar{F}}=\bar{F}_{i j} \boldsymbol{v}^{i j}=F_{k l} \boldsymbol{v}^{i j}=F_{23} \boldsymbol{v}^{01}-F_{13} \boldsymbol{v}^{02}+F_{12} \boldsymbol{v}^{03}+F_{03} \boldsymbol{v}^{12}-F_{02} \boldsymbol{v}^{13}+F_{01} \boldsymbol{v}^{23}
$$

\section{Vector Properties in $\mathbb{R}^{4,2}$}

\subsection{Conversion to Matrix Form Property}

3.1.1. Let $\vec{F}$ Be Any Vector in $\mathbb{R}^{4,2}$, It Can Be Written as the Main Matrix $F_{\mu y}$ in the Form

$(\vec{F}) \equiv F_{\mu \gamma}$, where

$$
F_{\mu \gamma}=\left(\begin{array}{llll}
F_{00} & F_{01} & F_{02} & F_{03} \\
F_{10} & F_{11} & F_{12} & F_{13} \\
F_{20} & F_{21} & F_{22} & F_{23} \\
F_{30} & F_{31} & F_{32} & F_{33}
\end{array}\right)
$$

3.1.2. The Dual Vector $\overrightarrow{\bar{F}}$, It Can Be Written as the Dual Matrix $\bar{F}_{\mu \gamma}$ in the Form

$(\overrightarrow{\bar{F}}) \equiv \bar{F}_{\mu \gamma}=F_{\rho \lambda}$, then 


$$
\bar{F}_{\mu \gamma}=\left(\begin{array}{llll}
F_{00} & F_{23} & F_{31} & F_{12} \\
F_{32} & F_{11} & F_{03} & F_{20} \\
F_{13} & F_{30} & F_{22} & F_{01} \\
F_{21} & F_{02} & F_{10} & F_{33}
\end{array}\right)
$$

\subsection{The Mix Product Property}

3.2.1. The Mix Cross-Product of Two Vectors $C \in \mathbb{R}^{4,1}$ and $\vec{F} \in \mathbb{R}^{4,2}$ Is Defined by Setting

$$
\begin{gathered}
\boldsymbol{C} \times \vec{F}=-\left(C^{\gamma} F_{\mu \gamma}\right) e^{\mu} \\
C^{\gamma} F_{\mu \gamma}=\left(\begin{array}{llll}
c^{0} & c^{1} & c^{2} & c^{3}
\end{array}\right)\left(\begin{array}{llll}
F_{00} & F_{01} & F_{02} & F_{03} \\
F_{10} & F_{11} & F_{12} & F_{13} \\
F_{20} & F_{21} & F_{22} & F_{23} \\
F_{30} & F_{31} & F_{32} & F_{33}
\end{array}\right) \\
=\left(\begin{array}{l}
c^{0} F_{00}+c^{1} F_{10}+c^{2} F_{20}+c^{3} F_{30} \\
c^{0} F_{01}+c^{1} F_{11}+c^{2} F_{21}+c^{3} F_{31} \\
c^{0} F_{02}+c^{1} F_{12}+c^{2} F_{22}+c^{3} F_{32} \\
c^{0} F_{03}+c^{1} F_{13}+c^{2} F_{23}+c^{3} F_{33}
\end{array}\right)
\end{gathered}
$$

3.2.2. The Mix Dot-Product of Two Vectors $C \in \mathbb{R}^{4,1}$ and $\vec{F} \in \mathbb{R}^{4,2}$ Is Defined by Setting

$$
\boldsymbol{C} \cdot \vec{F}=\boldsymbol{C} \times \overrightarrow{\bar{F}}=\boldsymbol{C} \times \boldsymbol{A} \times \boldsymbol{B}
$$

where, $\vec{F}=\boldsymbol{A} \times \boldsymbol{B}$.

From (2.1), we therefore get

$$
\begin{aligned}
\boldsymbol{C} \times \overrightarrow{\bar{F}}=-\left(C^{\gamma} \bar{F}_{\mu \gamma}\right) e^{\mu}=-\left(C_{\gamma} F_{\rho \lambda}\right) e^{\mu} \\
C^{\gamma} F_{\rho \lambda}=\left(\begin{array}{llll}
c_{0} & c_{1} & c_{2} & c_{3}
\end{array}\right)\left(\begin{array}{llll}
F_{00} & F_{23} & F_{31} & F_{12} \\
F_{32} & F_{11} & F_{03} & F_{20} \\
F_{13} & F_{30} & F_{22} & F_{01} \\
F_{21} & F_{02} & F_{10} & F_{33}
\end{array}\right) \\
=\left(\begin{array}{l}
c_{0} F_{00}+c_{1} F_{32}+c_{2} F_{13}+c_{3} F_{21} \\
c_{0} F_{23}+c_{1} F_{11}+c_{2} F_{30}+c_{3} F_{02} \\
c_{0} F_{31}+c_{1} F_{03}+c_{2} F_{22}+c_{3} F_{10} \\
c_{0} F_{12}+c_{1} F_{20}+c_{2} F_{01}+c_{3} F_{33}
\end{array}\right)
\end{aligned}
$$

Now in the example at hand, we have

$$
\begin{gathered}
F_{\mu \gamma}=\left(\begin{array}{llll}
F_{00} & F_{01} & F_{02} & F_{03} \\
F_{10} & F_{11} & F_{12} & F_{13} \\
F_{20} & F_{21} & F_{22} & F_{23} \\
F_{30} & F_{31} & F_{32} & F_{33}
\end{array}\right)=\left(\begin{array}{cccc}
0 & -76 & 51 & 46 \\
76 & 0 & 41 & 262 \\
-51 & -41 & 0 & -151 \\
-46 & -262 & 151 & 0
\end{array}\right) \\
\bar{F}_{\mu \gamma}=\left(\begin{array}{llll}
F_{00} & F_{23} & F_{31} & F_{12} \\
F_{32} & F_{11} & F_{03} & F_{20} \\
F_{13} & F_{30} & F_{22} & F_{01} \\
F_{21} & F_{02} & F_{10} & F_{33}
\end{array}\right)=\left(\begin{array}{cccc}
0 & -151 & -262 & 41 \\
151 & 0 & 46 & -51 \\
262 & -46 & 0 & -76 \\
-41 & 51 & 76 & 0
\end{array}\right)
\end{gathered}
$$


Let, $\boldsymbol{V}=4 e^{0}+9 e^{1}+23 e^{2}+4 e^{3}$.

Then, the cross-product of two vectors $V$ and $\vec{F}$ is defined as

$$
\begin{aligned}
V^{\gamma} F_{\mu \gamma} & =-\left(\begin{array}{llll}
4 & 9 & 23 & 4
\end{array}\right)\left(\begin{array}{cccc}
0 & -76 & 51 & 46 \\
76 & 0 & 41 & 262 \\
-51 & -41 & 0 & -151 \\
-46 & -262 & 151 & 0
\end{array}\right) \\
& =\left(\begin{array}{llll}
673 & 2295 & -1177 & 931
\end{array}\right)
\end{aligned}
$$

On the other hand, we have from vector relations the equation

$$
\begin{aligned}
\boldsymbol{V} \times \vec{F} & =\boldsymbol{V} \times(\boldsymbol{A} \times \boldsymbol{B})=\boldsymbol{A}(\boldsymbol{V} \cdot \boldsymbol{B})-\boldsymbol{B}(\boldsymbol{V} \cdot \boldsymbol{A}) \\
& =\left(5 e^{0}+7 e^{1}-2 e^{2}+13 e^{3}\right)(89)-\left(3 e^{0}-11 e^{1}+9 e^{2}+17 e^{3}\right)(188) \\
& =673 e^{0}+2295 e^{1}-1177 e^{2}+931 e^{3}
\end{aligned}
$$

Here the last vector equals the Equation (1).

The dot-product of $\boldsymbol{V}$ and $\vec{F}$ is defined as

$$
\begin{aligned}
V^{\gamma} \bar{F}_{\mu \gamma} & =-\left(\begin{array}{llll}
4 & 9 & 23 & 4
\end{array}\right)\left(\begin{array}{cccc}
0 & -151 & -262 & 41 \\
151 & 0 & 46 & -51 \\
262 & -46 & 0 & -76 \\
-41 & 51 & 76 & 0
\end{array}\right) \\
& =\left(\begin{array}{llll}
7221 & -1458 & -330 & -2043
\end{array}\right)
\end{aligned}
$$

From (3.2), we therefore get

$$
\begin{aligned}
\boldsymbol{V} \cdot \vec{F} & =\boldsymbol{C} \times \boldsymbol{A} \times \boldsymbol{B}=\left(\begin{array}{cccc}
e^{0} & e^{1} & e^{2} & e^{3} \\
4 & 9 & 23 & 4 \\
5 & 7 & -2 & 13 \\
3 & -11 & 9 & 17
\end{array}\right) \\
& =7221 e^{0}-1458 e^{1}-330 e^{2}-2043 e^{3}
\end{aligned}
$$

Here the new vector appearing on the right-hand side equals the Equation (II).

\section{The Force Equations on A.E Filed}

On A.E filed, there are only two types of forces namely cross and dot forces

\subsection{Calculate the Cross Force $F_{\text {cross }}$}

Let $\boldsymbol{F}_{\text {cross }}$ be 4 -force in the form

$$
\boldsymbol{F}_{\text {cross }}=\boldsymbol{v} \times \vec{F} \text { where } \vec{F}=m \vec{w}
$$

Therefore

$$
\begin{aligned}
\boldsymbol{v} \times \vec{F}=\boldsymbol{v} \times m \vec{w}=\boldsymbol{P} \times \vec{w} & \text { as } \\
\vec{w} & =w_{i j} \boldsymbol{v}^{i j}, \boldsymbol{P}=m \boldsymbol{v}_{i}=p_{i} e^{i}, i, j=0,1,2,3
\end{aligned}
$$

Then

$$
\boldsymbol{F}_{\text {cross }}=\boldsymbol{P} \times \vec{w}=\boldsymbol{p} \times\left(w_{0 i} v^{0 i}+w_{j k} \boldsymbol{k}^{j k}\right)=\boldsymbol{p} \times w_{0 i} v^{0 i}+\boldsymbol{p} \times w_{j k} \boldsymbol{k}^{j k}
$$

According to the three-orthogonal vectors $e^{1}, e^{2}, e^{3}$ we can rewrite the field 
vectors as

$$
w_{j k} v^{j k} \equiv \epsilon_{i j k} \omega_{i} e^{i}=\omega, i, j, k=1,2,3
$$

Now consider the equations

$$
\overline{\boldsymbol{\omega}}=\bar{\omega}_{i} e^{i} \text { and } w_{0 i}=\bar{\omega}_{i}, i=1,2,3
$$

Thus, the Equation (4.1) becomes

$$
\boldsymbol{F}_{\text {cross }}=\boldsymbol{p} \times\left(\boldsymbol{e}^{0} \times \overline{\boldsymbol{\omega}}\right)+\boldsymbol{p} \times \boldsymbol{\omega}
$$

we therefore get

$$
\boldsymbol{F}_{\text {cross }}=(\boldsymbol{p} \cdot \overline{\boldsymbol{\omega}}) \boldsymbol{e}^{0}-p_{0} \overline{\boldsymbol{\omega}}+\boldsymbol{p} \times \boldsymbol{\omega}
$$

\subsection{Calculate the Dot Force $\boldsymbol{F}_{\text {dot }}$}

Let $\boldsymbol{F}_{\mathrm{dot}}$ be 4 -Force in the form

$$
\boldsymbol{F}_{\mathrm{dot}}=\boldsymbol{v} \cdot \vec{F}
$$

From Equation (3.2) we have

$$
\boldsymbol{v} \cdot \vec{F}=\boldsymbol{v} \times \overrightarrow{\bar{F}}=\boldsymbol{v} \times m \overrightarrow{\bar{w}}=\boldsymbol{P} \times \overrightarrow{\bar{w}}
$$

where

$$
\overrightarrow{\bar{F}}=m \overrightarrow{\bar{w}}, \overrightarrow{\bar{w}}=\bar{w}_{i j} \boldsymbol{v}^{i j}, \boldsymbol{P}=p_{i} e^{i} \text { with } i, j=0,1,2,3
$$

Using the three-orthogonal vectors $e^{1}, e^{2}, e^{3}$ we can rewrite the transformation in Equation (4.2) and Equation (4.3) as

$$
w_{j k}=\omega_{i}, w_{0 i}=\bar{\omega}_{i}, i, j, k=1,2,3
$$

then we obtain

$$
\vec{w}_{\mu \gamma}=\left(\begin{array}{cccc}
w_{00} & w_{23} & w_{31} & w_{12} \\
w_{32} & w_{11} & w_{03} & w_{20} \\
w_{13} & w_{30} & w_{22} & w_{01} \\
w_{21} & w_{02} & w_{10} & w_{33}
\end{array}\right)=\left(\begin{array}{cccc}
0 & \omega_{1} & \omega_{2} & \omega_{3} \\
-\omega_{1} & 0 & \bar{\omega}_{3} & -\bar{\omega}_{2} \\
-\omega_{2} & -\bar{\omega}_{3} & 0 & \bar{\omega}_{1} \\
-\omega_{3} & \bar{\omega}_{2} & -\bar{\omega}_{1} & 0
\end{array}\right)
$$

Thus

$$
\begin{aligned}
\boldsymbol{F}_{\mathrm{dot}}= & -\left(\begin{array}{llll}
p_{0} & p_{1} & p_{2} & p_{3}
\end{array}\right)\left(\begin{array}{cccc}
0 & \omega_{1} & \omega_{2} & \omega_{3} \\
-\omega_{1} & 0 & \bar{\omega}_{3} & -\bar{\omega}_{2} \\
-\omega_{2} & -\bar{\omega}_{3} & 0 & \bar{\omega}_{1} \\
-\omega_{3} & \bar{\omega}_{2} & -\bar{\omega}_{1} & 0
\end{array}\right) \\
= & -\left\{-\left(0+p_{1} \omega_{1}+p_{2} \omega_{2}+p_{3} \omega_{3}\right) e^{0}+\left(p_{0} \omega_{1}+0-p_{2} \bar{\omega}_{3}+p_{3} \bar{\omega}_{2}\right) e^{1}\right. \\
& \left.+\left(p_{0} \omega_{2}+p_{1} \bar{\omega}_{3}+0-p_{3} \bar{\omega}_{1}\right) e^{2}+\left(p_{0} \omega_{3}-p_{1} \bar{\omega}_{2}+p_{2} \bar{\omega}_{1}+0\right) e^{3}\right\} \\
= & -\left\{-\left(p_{1} \omega_{1}+p_{2} \omega_{2}+p_{3} \omega_{3}\right) e^{0}+\left(\omega_{1} e^{1}+\omega_{2} e^{2}+\omega_{3} e^{3}\right) p_{0}\right. \\
& \left.-\left[\left(p_{2} \bar{\omega}_{3}-p_{3} \bar{\omega}_{2}\right) e^{1}-\left(p_{1} \bar{\omega}_{3}-p_{3} \bar{\omega}_{1}\right) e^{2}+\left(p_{1} \bar{\omega}_{2}-p_{2} \bar{\omega}_{1}\right) e^{3}\right]\right\}
\end{aligned}
$$

For the orthogonal unit vectors $e^{1}, e^{2}, e^{3}$ the last equation becomes

$$
\boldsymbol{F}_{\mathrm{dot}}=(\boldsymbol{p} \cdot \boldsymbol{\omega}) e^{0}-p^{0} \boldsymbol{\omega}+\boldsymbol{p} \times \overline{\boldsymbol{\omega}}
$$


where

$$
\overline{\boldsymbol{\omega}}=\bar{\omega}_{i} e^{i}, \boldsymbol{\omega}=\omega_{i} e^{i} \text { with } i=1,2,3
$$

\section{Let in our example}

$$
\boldsymbol{P}=4 e^{0}+9 e^{1}+23 e^{2}+4 e^{3}: \overrightarrow{\boldsymbol{w}}=\boldsymbol{A} \times \boldsymbol{B}
$$

Then

$$
\begin{gathered}
\boldsymbol{p}^{0}=4 e^{0}, \quad \boldsymbol{p}=9 e^{1}+23 e^{2}+4 e^{3} \\
\boldsymbol{\omega}=-151 e^{1}-262 e^{2}+41 e^{3} \\
\overline{\boldsymbol{\omega}}=-76 e^{1}+51 e^{2}+46 e^{3}
\end{gathered}
$$

thus from Equation (4.4) and Equation (4.5) we get

$$
\begin{aligned}
\boldsymbol{F}_{\text {cross }} & =\boldsymbol{v} \times \vec{F}=(\boldsymbol{p} \cdot \overline{\boldsymbol{\omega}}) e^{0}-p_{0} \overline{\boldsymbol{\omega}}+\boldsymbol{p} \times \boldsymbol{\omega} \\
& =673 e^{0}+2295 e^{1}-1177 e^{2}+931 e^{3} \\
\boldsymbol{F}_{\mathrm{dot}}= & \boldsymbol{v} \cdot \vec{F}=(\boldsymbol{p} \cdot \boldsymbol{\omega}) e^{0}-p^{0} \boldsymbol{\omega}+\boldsymbol{p} \times \overline{\boldsymbol{\omega}} \\
= & -7221 e^{0}+1458 e^{1}+330 e^{2}+2043 e^{3}
\end{aligned}
$$

The last two equations equals the Equation (I) and the Equation (II).

\section{The Relationships between the Force Equations on A.E Filed and the Conventional Force}

\subsection{Calculate the Conventional Ordinary Force}

The 4-momentum $\boldsymbol{P}$ of a particle of mass $m_{0}$ at position $\boldsymbol{r}=\{t, \tilde{\boldsymbol{r}}\}$ moving at velocity $\boldsymbol{V}=\frac{\mathrm{d} \boldsymbol{r}}{\mathrm{d} \tau}$ can be written as

$$
\boldsymbol{P}=m_{0} \boldsymbol{V}=m_{0} \frac{\mathrm{d}\{t, \tilde{\boldsymbol{r}}\}}{\mathrm{d} \tau}=m_{0}\left(\frac{\mathrm{d} t}{\mathrm{~d} \tau}, \frac{\mathrm{d} \tilde{\boldsymbol{r}}}{\mathrm{d} \tau}\right)=m_{0}(c \gamma, \gamma \boldsymbol{v})=(m c, m \boldsymbol{v})=\left(p_{0}, \boldsymbol{p}\right)
$$

The 3-velocity $\boldsymbol{v}$ of the particle is defined by $\boldsymbol{v}=\frac{\mathrm{d} \tilde{\boldsymbol{r}}}{\mathrm{d} \tau}=v_{i} e^{i}+\boldsymbol{\omega} \times \tilde{\boldsymbol{r}}$, then

$$
\boldsymbol{p}=m \boldsymbol{v}=m v_{i} e^{i}+m(\boldsymbol{\omega} \times \tilde{\boldsymbol{r}})=\left(p_{i}+p_{i}\right) e^{i}
$$

where $\omega$ is called an angular velocity vector of the rotating system,

$$
\boldsymbol{\omega}=\frac{1}{2 m}(\nabla \times p)
$$

$\boldsymbol{p}$ is the 4-momentum of the coordinate system itself, $\boldsymbol{p}=\left(\boldsymbol{\partial}_{0}, \boldsymbol{p}\right)$

Thus

$$
\begin{aligned}
\frac{\partial \boldsymbol{P}}{\partial t}=\frac{\partial \boldsymbol{p}_{0}}{\partial t} & +\frac{\partial \boldsymbol{p}}{\partial t}=\frac{\partial m c}{\partial t} e^{0}+\left(\partial_{t} p_{i}+\partial_{t} \not \partial_{i}\right) e^{i}+\boldsymbol{\omega} \times \boldsymbol{p} \\
\left(\partial_{t} p_{i}\right) e^{i} & =\partial_{t} \boldsymbol{P}-\frac{\partial m c}{\partial t} e^{0}-\left(\partial_{t} \not \partial_{i}\right) e^{i}-\boldsymbol{\omega} \times \boldsymbol{p} \\
& =-\frac{\partial m c}{\partial t} e^{0}+\left(\partial_{t} P_{i}-\partial_{t} \partial_{i}\right) e^{i}+\boldsymbol{p} \times \boldsymbol{\omega}
\end{aligned}
$$

Now, we can rewrite last equation as the following

$$
\overline{\boldsymbol{F}}^{\prime}=\boldsymbol{f}^{\theta}+\overline{\boldsymbol{F}}+\boldsymbol{p} \times \boldsymbol{\omega}
$$


where

$$
\begin{aligned}
\overline{\boldsymbol{F}}^{\prime} & =\frac{\partial \boldsymbol{p}}{\partial t}: \boldsymbol{f}^{\theta}=-\frac{\partial m c}{\partial t} e^{0} \\
\overline{\boldsymbol{F}} & =\left(\partial_{t} P_{i}-\partial_{t} \partial_{i}\right) e^{i}
\end{aligned}
$$

\subsection{Comparison to Cross Force}

If the Equation (5.1) is equivalent to the cross force equations in (4.4), we shall have

$$
\overline{\boldsymbol{F}}=-p_{0} \overline{\boldsymbol{\omega}}, \boldsymbol{f}^{\theta}=(\boldsymbol{p} \cdot \overline{\boldsymbol{\omega}}) e^{0}
$$

\section{Some Special Results}

\subsection{Covariant Conventional Force}

From comparison above, we have $\overline{\boldsymbol{F}}^{\prime}=\boldsymbol{F}_{\text {cross }}$, thus

$$
\begin{gathered}
\overline{\boldsymbol{F}}^{\prime}=\frac{\partial \boldsymbol{p}}{\partial t}=\boldsymbol{P} \times \overrightarrow{\boldsymbol{w}} \\
\bar{F}_{\mu}^{\prime}=-P^{\gamma} w_{\mu \gamma}=w_{\mu \gamma} P^{\gamma}=\bar{F}_{\mu \gamma} v^{\gamma}
\end{gathered}
$$

\subsection{The Value of the Component $f o$}

From Equation (5.3) then,

$$
\boldsymbol{f}^{\theta}=(\boldsymbol{p} \cdot \overline{\boldsymbol{\omega}})=-\frac{1}{p_{0}}(\boldsymbol{p} \cdot \overline{\boldsymbol{F}})=-\left(\frac{\boldsymbol{v}}{c} \cdot \overline{\boldsymbol{F}}\right)
$$

\subsection{The Equation of 4-Angular Velocity $\vec{w}$}

Return above we have in the three-dimensional space

$$
w_{j k} \boldsymbol{v}^{j k} \equiv \boldsymbol{\omega}=\frac{1}{2 m}(\nabla \times p), \quad j, k=1,2,3
$$

So, in the four-dimensional space time we Consider the $\vec{w}$ and $\omega$ components are given by $\vec{w}=\frac{1}{m}(\nabla \times P) \nabla$ bold line, $\boldsymbol{\omega}=\frac{1}{m}(\nabla \times p) \nabla$ normal line, where $\nabla=\left(\frac{\partial}{\partial x_{0}}, \nabla\right)$.

For E M case, Let $A$ is the vector potential and $p=q A[1]$ then we get

$$
\begin{aligned}
& \vec{w}=\frac{1}{m}(\nabla \times p)=\frac{q}{m}(\nabla \times \boldsymbol{A})=\frac{q}{m} \vec{B} \\
& \boldsymbol{\omega}=\frac{1}{m}(\nabla \times p)=\frac{q}{m}(\nabla \times \boldsymbol{A})=\frac{q}{m} \boldsymbol{B}
\end{aligned}
$$

\subsection{The Value of $p_{0}$}

The sub determinant of angular velocity $w_{0 i}$ is defined by

$$
w_{0 i}=\frac{1}{m}\left(\partial_{0} \partial_{i}-\partial_{i} \partial_{0}\right) \quad i=1,2,3
$$


From Equation (5.3) and Equation (4.3) we then get

$$
\bar{F}=-p_{0} \bar{\omega}=-p_{0} w_{0 i} e^{i}=-c\left(\partial_{0} \partial_{i}-\partial_{i} \partial_{0}\right) e^{i}=-\left(\partial_{t} \partial_{i}-c \partial_{i} \partial_{0}\right) e^{i}
$$

which is equivalent to Equation (5.2).

$$
\begin{gathered}
-\left(\partial_{t} \partial_{i}-c \partial_{i} \partial_{0}\right)=\left(\partial_{t} P_{i}-\partial_{t} \partial_{i}\right) \\
c \partial_{i} \partial_{0}=\partial_{t} P_{i}=-\partial_{i} \varnothing \\
\partial_{0}=-\frac{\emptyset}{c}
\end{gathered}
$$

where $\emptyset$ is scalar potential energy, so we can write the 4 -coordinate momentum $p$ as,

$$
p^{2}=\left(\partial_{0}, \partial_{i}\right)=\left(-\frac{\emptyset}{c}, \partial_{i}\right)
$$

For 4-vector potential $\boldsymbol{A}$, we get [2]

$$
\boldsymbol{A}=\left(A_{0}, A_{i}\right)=\left(-\frac{\varnothing}{c}, A_{i}\right)
$$

\subsection{Calculate the Dual to $F^{\prime}$}

We suppose that the dual force $\overline{\boldsymbol{F}}^{\prime}$ is defined by Equation (4.5) as the following

$$
\boldsymbol{F}^{\prime}=\boldsymbol{f}^{0}+\boldsymbol{F}+\boldsymbol{p} \times \overline{\boldsymbol{\omega}}
$$

where

$$
\boldsymbol{F}^{\prime}=\boldsymbol{F}_{\mathrm{dot}}, \boldsymbol{f}^{0}=(\boldsymbol{p} \cdot \boldsymbol{\omega}) e^{0}, \boldsymbol{F}=-p^{0} \boldsymbol{\omega}
$$

\section{Conclusion}

In A.E space, all force equations [3] (e.g. Coriolis Force, Lorentz force, ordinary force, Maxwell's Equations and others) are elegantly represented by two simple equations

$$
\boldsymbol{F}_{\text {cross }}=\boldsymbol{c} \times \vec{F}, F_{d o t}=\boldsymbol{c} \cdot \vec{F} \text { where } \vec{F}=m \vec{w}, \boldsymbol{c}=\boldsymbol{v} \text { or } \boldsymbol{\nabla}
$$

\section{Discussion}

\subsection{E M Field Tenors}

Using the transformation in Equation (4.2) and Equation (4.3), we obtain

$$
w_{\mu \gamma}=\left(\begin{array}{llll}
w_{00} & w_{01} & w_{02} & w_{03} \\
w_{10} & w_{11} & w_{12} & w_{13} \\
w_{20} & w_{21} & w_{22} & w_{23} \\
w_{30} & w_{31} & w_{32} & w_{33}
\end{array}\right)=\left(\begin{array}{cccc}
0 & \bar{\omega}_{1} & \bar{\omega}_{2} & \bar{\omega}_{3} \\
-\bar{\omega}_{1} & 0 & \omega_{3} & -\omega_{2} \\
-\bar{\omega}_{2} & -\omega_{3} & 0 & \omega_{1} \\
-\bar{\omega}_{3} & \omega_{2} & -\omega_{1} & 0
\end{array}\right)
$$

The Equation (6.3.1) and Equation (6.3.2) follow that

$$
w_{\mu \gamma}=\frac{q}{m} B_{\mu \gamma}: \omega_{i}=(\omega)_{i}=\frac{q}{m} B_{i}
$$

By assuming that $\overline{\boldsymbol{F}}=q \boldsymbol{E}$, then from Equation (5.3) we get 


$$
\bar{\omega}_{i}=-\frac{1}{p_{0}} \bar{F}_{i}=-\frac{q}{m} \frac{E_{i}}{c}, i=1,2,3
$$

and so on. The overall result is [2]

$$
B_{\mu \gamma}=\left(\begin{array}{cccc}
0 & -\frac{E_{1}}{c} & -\frac{E_{2}}{c} & -\frac{E_{3}}{c} \\
\frac{E_{1}}{c} & 0 & B_{3} & -B_{2} \\
\frac{E_{2}}{c} & -B_{3} & 0 & B_{1} \\
\frac{E_{3}}{c} & B_{2} & -B_{1} & 0
\end{array}\right)
$$

By a similar argument, we can write the dual matrix as

$$
E_{\mu \gamma}=\left(\begin{array}{cccc}
0 & -B_{1} & -B_{2} & -B_{3} \\
B_{1} & 0 & \frac{E_{3}}{c} & -\frac{E_{2}}{c} \\
B_{2} & -\frac{E_{3}}{c} & 0 & \frac{E_{1}}{c} \\
B_{3} & \frac{E_{2}}{c} & -\frac{E_{1}}{c} & 0
\end{array}\right)
$$

\subsection{Lorentz Force Law [4]}

\subsubsection{The First Force Equations on A.E Filed}

Without the component $\boldsymbol{f}^{\theta}$, the Equation (5.1) becomes

$$
\overline{\boldsymbol{F}}^{\prime}=\boldsymbol{P} \times \vec{w}=\overline{\boldsymbol{F}}+\boldsymbol{p} \times \boldsymbol{\omega}
$$

to get the first E M Lorentz force law. Let

$$
\overline{\boldsymbol{F}}^{\prime}=q \boldsymbol{E}^{\prime}: \overline{\boldsymbol{F}}=q \boldsymbol{E}: \vec{w}=\frac{q}{m} \vec{B} \text { and } \boldsymbol{\omega}=\frac{q}{m} \boldsymbol{B}
$$

It follows that

$$
\begin{gathered}
q \boldsymbol{E}^{\prime}=\boldsymbol{p} \times \frac{q}{m} \vec{B}=q \boldsymbol{E}+m \boldsymbol{v} \times \frac{q}{m} \boldsymbol{B} \\
\boldsymbol{E}^{\prime}=\boldsymbol{V} \times \vec{B}=\boldsymbol{E}+\boldsymbol{v} \times \boldsymbol{B}
\end{gathered}
$$

\subsubsection{The Second Force Equations on A.E Filed}

Without the component $\boldsymbol{f}^{\theta}$, the the Equation (6.4) becomes

$$
\boldsymbol{F}^{\prime}=\boldsymbol{P} \times \overrightarrow{\bar{w}}=\boldsymbol{F}+\boldsymbol{p} \times \overline{\boldsymbol{\omega}}
$$

to get the second E M Lorentz force law. Let

$$
\begin{gathered}
\boldsymbol{F}^{\prime}=q \boldsymbol{H}^{\prime}=q \frac{1}{\mu_{0}} \boldsymbol{B}^{\prime}: \boldsymbol{F}=q \boldsymbol{H}=q \frac{1}{\mu_{0}} \boldsymbol{B} \\
\overrightarrow{\vec{w}}=\frac{q}{m} \vec{D}=\frac{q}{m} \epsilon_{0} \vec{E} \text { and } \overline{\boldsymbol{\omega}}=\frac{q}{m} \boldsymbol{D}=\frac{q}{m} \epsilon_{0} \boldsymbol{E}
\end{gathered}
$$

It follows that 


$$
\begin{gathered}
q \frac{1}{\mu_{0}} \boldsymbol{B}^{\prime}=m \boldsymbol{V} \times \frac{q}{m} \epsilon_{0} \vec{E}=q \frac{1}{\mu_{0}} \boldsymbol{B}+m \boldsymbol{v} \times \frac{q}{m} \epsilon_{0} \boldsymbol{E} \\
\boldsymbol{B}^{\prime}=\frac{\boldsymbol{V}}{c^{2}} \times \vec{E}=B+\frac{\boldsymbol{v}}{c^{2}} \times \boldsymbol{E}
\end{gathered}
$$

\subsection{The 4-Field Equations in Tensor Notation [2]}

According to the equations above, we can define 4-Maxwell's Equations by suppose that

$$
c=\nabla, \vec{F}=q \vec{B}, \vec{B}=\nabla \times \boldsymbol{A}
$$

\subsubsection{The Inhomogeneous E M Maxwell's Equations}

By vector triple product we have

$$
\begin{aligned}
& \boldsymbol{c} \times \vec{F}=\nabla \times q \vec{B}=q \nabla \times(\nabla \times \boldsymbol{A})=q(\nabla \cdot \boldsymbol{A}) \nabla-q(\nabla \cdot \nabla) \boldsymbol{A}=-q \nabla^{2} \boldsymbol{A}=q \boldsymbol{J}=q J_{\gamma} e^{\gamma} \\
& \text { But } \\
& \nabla \times q \vec{B}=-q\left(\partial^{\mu} B_{\gamma \mu}\right) e^{\gamma} \text {, therefore } \\
& \qquad \partial^{\mu} B_{\gamma \mu}=-J_{\gamma} \Leftrightarrow \partial^{0} B_{0 j}+\partial^{i} B_{i j}=J_{j}
\end{aligned}
$$

\subsubsection{The Homogeneous Equations}

$$
c \cdot \vec{F}=\nabla \cdot \vec{F}=\nabla \times \vec{F}=-\left(\partial^{\gamma} \bar{F}_{\mu \gamma}\right) e^{\mu}
$$

From Equation (3.3) thus

$\nabla \cdot \vec{F}=-\left(\partial_{\gamma} F_{\rho \lambda}\right) e^{\mu}=-q\left(\partial_{\gamma} B_{\rho \lambda}\right) e^{\mu}$, but

$\nabla \cdot \vec{F}=\nabla \cdot q \vec{B}=q \nabla \cdot(\nabla \times \boldsymbol{A})=q(\nabla \times \nabla \times \boldsymbol{A})=0$, then

$$
\partial_{\gamma} B_{\rho \lambda}=0 \Leftrightarrow \partial_{\gamma} B_{\rho \lambda}+\partial_{\rho} B_{\lambda \gamma}+\partial_{\lambda} B_{\gamma \rho}
$$

\section{Acknowledgements}

I have named the new space in this paper as A.E (Abou Layla-Erdogan's) as an expression of my thanks and appreciation for the Turkish President's humanitarian attitudes towards my people and appreciation for my Turkish friends that supported me during my high study in Turkey.

\section{References}

[1] Abou Layla, A.K. (2017) Calclation the Exact Value of Gravitational Constant. LAP LAMBERT Academic Publishing. https://www.amazon.com/dp/3330337257/ref=cm_sw_r_fa_dp_U_-DfZAb9WVV8QK

[2] Blau, M. (2017) Lecture Notes on General Relativity. http://www.blau.itp.unibe.ch/newlecturesGR.pdf

[3] Abou Layla, A.K. (2017) Symmetry in Equations of Motion between the Atomic and Astronomical Models. Journal of High Energy Physics, Gravitation and Cosmology, 3, 328-338. http://www.scirp.org/journal/PaperDownload.aspx?paperID=75700 https://doi.org/10.4236/jhepgc.2017.32028

[4] Wikipedia. Lorentz force. https://en.wikipedia.org/wiki/Lorentz_force 